\section{Beat the pain of sensitivity}

Arm \& Hammer has developed Sensitive Pro Repair toothpaste specifically to address the cause of sensitive teeth. This specially formulated toothpaste contains ingredients that have been clinically proven to dramatically reduce dentine hypersensitivity. Plus, it is powered by the patented Arm \& Hammer Liquid Calcium technology.

Used regularly for eight weeks, Sensitive Pro Repair toothpaste offers lasting relief from dentine hypersensitivity for up to 16 weeks.

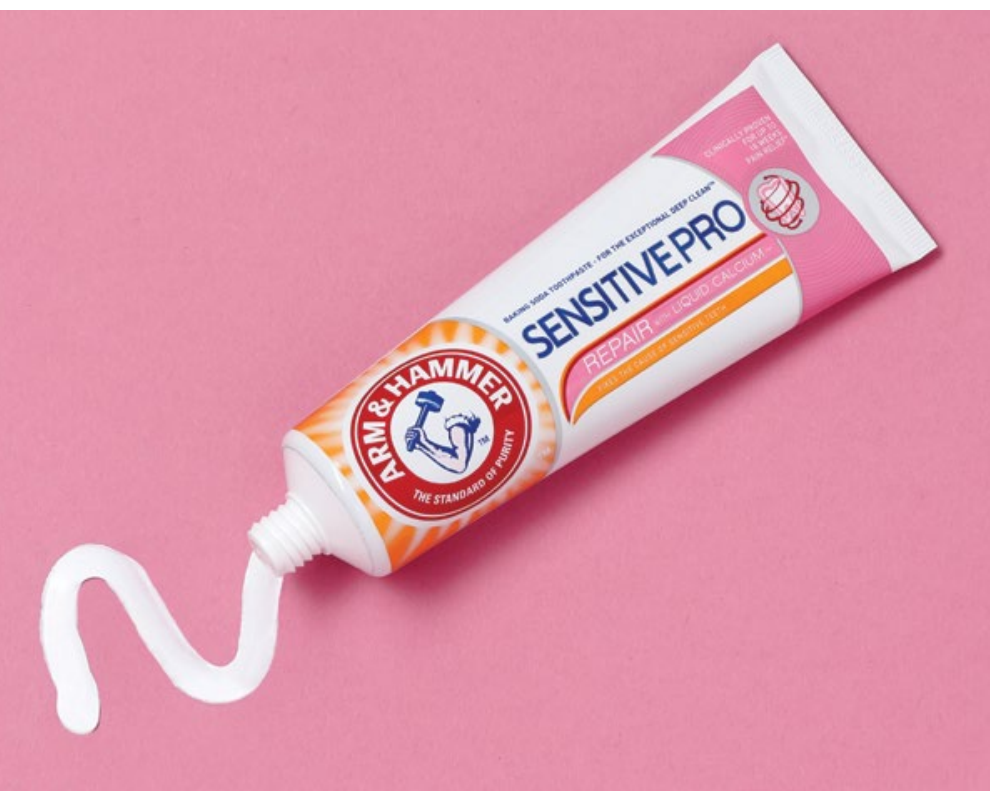

For more information, visit http://www.armandhammer.co.uk/ or email: ukenquiries@churchdwight.com. Arm \& Hammer oral healthcare products are available at Boots, Superdrug, Sainsbury's, Tesco, Asda and Morrisons throughout the UK.

\section{Digital dentistry facilitated by optical 3D printers}

Since 2012 MiiCraft have developed optical 3D printers; their 3D printers have been used in dental labs for many years. MiiCraft's latest desktop model Prime 150 series is suitable for small and medium size dental labs and clinics. It can print $4 \mathrm{~cm}$ in height per hour, while the printing area is 150 $\mathrm{mm} \times 84 \mathrm{~mm}$ at 55 microns accuracy; in terms of output that is four dental arches in 30 minutes.

MiiCraft's standalone model Advance series is suitable for large dental labs; the printing area is $255 \mathrm{~mm}$ x $235 \mathrm{~mm}$ : 14 dental arches in 30 minutes.

All MiiCraft 3D printers are open system. Users can use the photopolymer resins MiiCraft provide, or any other photopolymer resins on the market, such as Detax, Nextdent and Keystone, etc. All world leading brands are compatible with MiiCraft 3D printers. One MiiCraft 3D printer can print tens of dental applications, including surgical guides, indirect bonding trays, model/arch, aligners, temporary dentures, castable frameworks, crowns and bridges, etc. Visit www.miicraft.com for more information or email sales@miicraft.com.

\title{
Toothpaste and mouthwash inactivate $99.9 \%$ of the virus that causes COVID-19
}

Laboratory studies show that toothpastes containing zinc or stannous and mouthwash formulas with cetylpyridinium chloride (CPC) neutralise the virus that causes COVID-19 by $99.9 \%$.

The studies are part of a Colgate research programme that includes clinical studies among infected people to assess the efficacy of oral care products in reducing the amount of the virus in the mouth, potentially slowing the transmission of the COVID-19 virus.

In the laboratory studies - the first to include toothpaste Colgate Total and Meridol toothpaste neutralised $99.9 \%$ of the virus after two minutes of contact. Colgate Plax, Colgate Total and Colgate Zero mouthwashes were similarly effective after 30 seconds. The studies, completed in October, were conducted in partnership with Rutgers University's Public Health Research Institute and Regional Biosafety Laboratories.

The results suggest that some toothpastes and mouthwashes may help reduce the spread of SARS-CoV-2, the virus that causes COVID-19, by temporarily reducing the amount of virus in the mouth. The virus spreads through respiratory droplets or small particles produced when an infected person coughs, sneezes, sings, talks, or breathes, according to the US Centers for Disease Control and Prevention.

Concurrent to the laboratory study, a Colgate-sponsored clinical study involving some 50 hospitalised subjects with COVID-19 was conducted at the Albert Einstein Institute in Sao Paulo, Brazil. This study demonstrated the ability of certain Colgate mouthwashes to substantially reduce the amount of the virus in the mouth temporarily. The researchers plan to share their findings in early December. Additional Colgate-supported clinical research studies on toothpaste and mouthwashes are in early stages at Rutgers, the Einstein Institute, and at the University of North Carolina Adams School of Dentistry, with some 260 people with COVID-19 participating in these studies.

As the world's \# 1 trusted dental expert, Colgate is committed to leading in science and to ensuring that its products address health challenges and meet consumers' needs. For more information about the effects of oral hygiene on overall health and additional insights on mask mouth and other topics, visit www.colgate.com. 